M. OHTA

KODAI MATH. J.

18 (1995), 68-74

\title{
STABILITY AND INSTABILITY OF STANDING WAVES FOR ONE DIMENSIONAL NONLINEAR SCHRÖDINGER EQUATIONS WITH DOUBLE POWER NONLINEARITY
}

\author{
MASAHITO OHTA
}

\section{Introduction and main results}

In the present paper we consider the stability and instability of standing waves for the following nonlinear Schrödinger equation:

$$
i u_{t}+u_{x x}+f(u)=0, \quad t \geqq 0, x \in \boldsymbol{R},
$$

where $f(u)=a|u|^{p-1} u+b|u|^{q-1} u$ with $a, b \in \boldsymbol{R}$ and $1<p<q<\infty$.

Equation (1.1) arises in various regions of mathematical physics. For example, when $a>0, b<0, p=3$ and $q=5$, this equation appears in boson gas interaction, nonlinear optics, and so on (see, e.g., [1] and its references).

By a standing wave, we mean a solution of (1.1) with the form

$$
u(t, x)=e^{\imath \omega t} \phi_{\omega}(x),
$$

where $\omega>0$ and $\phi_{\omega}$ is a solution of the following problem:

$$
\left\{\begin{array}{l}
-\phi_{x x}+\omega \phi-f(\phi)=0, \quad x \in \boldsymbol{R}, \\
\phi \in H^{1}(\boldsymbol{R}), \quad \phi \neq 0 .
\end{array}\right.
$$

The existence and uniqueness of the solution of (1.2) are well known. Put $\omega^{*}=\sup \left\{\omega>0:(\omega / 2) s^{2}-F(s)<0\right.$ for some $\left.s>0\right\}$, where $F(s)=\int_{0}^{s} f(\sigma) d \sigma$. Then, for any $\omega \in\left(0, \omega^{*}\right)$, there exists a unique solution $\phi_{\omega}$ of (1.2), up to a translation and a phase change (see, e.g., [3]).

Stability and instability of standing waves are defined as follows.

DEFINITION. We shall say that the standing wave $u_{\omega}(t)=e^{2 \omega t} \phi_{\omega}$ is stable if for any $\varepsilon>0$ there exists $\delta>0$ with the following property: if $u_{0} \in H^{1}(\boldsymbol{R})$, $\left\|u_{0}-\phi_{\omega}\right\|_{H^{1}}<\delta$ and $u(t)$ is a solution of (1.1) with $u(0)=u_{0}$, then

$$
\sup _{0 \leqq t<\infty} \inf _{\theta, y \in R}\left\|u(t)-e^{i \theta} \tau_{y} \phi_{\omega}\right\|_{H 1}<\varepsilon,
$$

Received December 16, 1993. 
where $\tau_{y} v(x)=v(x-y)$. Otherwise, $u_{\omega}$ is said to be unstable.

The unique local existence theorem in $H^{1}(\boldsymbol{R})$ for (1.1) is already established: for any $u_{0} \in H^{1}(\boldsymbol{R})$, then there exist $T>0$ and a unique solution $u(\cdot) \in C([0, T)$; $\left.H^{1}(\boldsymbol{R})\right)$ of $(1.1)$ with $u(0)=u_{0}$ such that either $T=\infty$ or $T<\infty$ and $\lim _{t \rightarrow T}\|u(t)\|_{H^{1}}$ $=\infty$. Furthermore, $u(t)$ satisfies the two conservation laws $\|u(t)\|_{L^{2}}=\left\|u_{0}\right\|_{L^{2}}$ and $E(u(t))=E\left(u_{0}\right)$, where $E(v)=1 / 2\left\|v_{x}\right\|_{L^{2}}-\int_{-\infty}^{\infty} F(|v(x)|) d x$. For the details, see, e. g., [5], [6] and [10].

Recently, many authors have been studying the problem of stability and instability of standing waves for nonlinear Schrödinger equations (see, e.g., [2, $4,8,9,12,13]$ ).

In the single power case $f(u)=a|u|^{p-1}$ with $a>0$ and $p>1$, it is well known that if $1<p<5$, then $u_{\omega}$ is stable for any $\omega \in(0, \infty)$ (see [4]), and if $p \geqq 5$, then $u_{\omega}$ is unstable for any $\omega \in(0, \infty)$ (see [2] for $p>5$ and [13] for $p=5$ ).

In the double power case $(a \neq 0$ and $b \neq 0),(1.1)$ has no scaling invariance, while it exists in the single power case. The scaling invariance makes the stability and instability problem simple in the single power case (see [12]). However, we can not use the scaling argument in the double power case. This is why the double power case has not been studied as well as the single power case, regardless of its physical importance. In fact, the phenomena occurring in the double power case are quite different from those in the single power case (see Remarks $1 \sim 4$ below).

Our main results are the followings.

THEOREM 1. Let $a>0, b>0$ and $1<p<q<\infty$.

(1) If $q \leqq 5$, then $u_{\omega}$ is stable for any $\omega \in(0, \infty)$.

(2) If $p \geqq 5$, then $u_{\omega}$ is unstable for any $\omega \in(0, \infty)$.

(3) If $p<5<q$, then there exist positive constants $\omega_{1}$ and $\omega_{2}$ such that $u_{\omega}$ is stable for any $\omega \in\left(0, \omega_{1}\right)$, and unstable for any $\omega \in\left(\omega_{2}, \infty\right)$.

THEOREM 2. Let $a<0, b>0$ and $1<p<q<\infty$.

(1) If $q \geqq 5$, then $u_{\omega}$ is unstable for any $\omega \in(0, \infty)$.

(2) If $q<5$, then there exists a positive constant $\omega_{3}$ such that $u_{\omega}$ is stable for any $\omega \in\left(\omega_{3}, \infty\right)$.

(3) If $q<5$ and $p+q>6$, then there exists a positive constant $\omega_{4}$ such that $u_{\omega}$ is unstable for any $\omega \in\left(0, \omega_{4}\right)$.

THEOREM 3. Let $a>0, b<0$ and $1<p<q<\infty$.

(1) If $p \leqq 5$, then $u_{\omega}$ is stable for any $\omega \in\left(0, \omega^{*}\right)$.

(2) If $p>5$, then there exist positive constants $\omega_{5}$ and $\omega_{6}$ such that $u_{\omega}$ is unstable for any $\omega \in\left(0, \omega_{5}\right)$, and stable for any $\omega \in\left(\omega_{6}, \omega^{*}\right)$.

Remark 1. Recall that when $f(u)=a|u|^{p-1} u$ with $a>0$ and $p>1$, the critical 
exponent $p$ is 5 , that is, if $1<p<5$, then $u_{\omega}$ is stable for any $\omega \in(0, \infty)$, and if $p \geqq 5$, then $u_{\omega}$ is unstable for any $\omega \in(0, \infty)$. Note that in the single power case, the stability and instability of standing waves are determined only by the exponent $p$ and do not depend on the frequency $\omega$. Theorems $1(3), 2(2),(3)$ and 3 (2) are, to our knowledge, the first examples that admit both stable and unstable frequencies $\omega$ of standing waves for nonlinear Schrödinger equations.

Remark 2. It is well known that if a function $f:[0, \infty) \rightarrow \boldsymbol{R}$ satisfies the following condition $(\mathrm{G})$, we call it Glassey's condition, the solution $u(t)$ of $(1.1)$ with $u(0)=u_{0}$ blows up in a finite time if $E\left(u_{0}\right)<0$ and $\int_{-\infty}^{\infty}|x|^{2}\left|u_{0}(x)\right|^{2} d x<\infty$ (see $[7]):(G)$ There exists a constant $\gamma \geqq 6$ such that $s f(s) \geqq \gamma F(s)$ for all $s \geqq 0$. When $a>0, b>0$ and $p<5<q$, Glassey's condition does not hold, but Kurata and Ogawa [11] showed the existence of finite time blowing-up solutions of (1.1). It is a very interesting problem to investigate the relations between the instability of standing waves and the existence of finite time blowing-up solutions (see [13]).

Remark 3. In the single power case, $f(u)=b|u|^{4} u$ with $b>0, u_{\omega}$ is unstable for any $\omega \in(0, \infty)$. It seems natural to conjecture that $u_{\omega}$ is more unstable in the case of $f(u)=a|u|^{p-1} u+b|u|^{4} u$ with $a>0, b>0$ and $1<p<5$ than that of $f(u)=b|u|^{4} u$ with $b>0$. Nevertheless, Theorem 1 (1) shows that it is not so. Furthermore, when $a=4, b=3, p=3$ and $q=5$, we can show that $E\left(\phi_{\omega}\right)=$ $\tan ^{-1}((\sqrt{1+\omega}-1) / \sqrt{\omega})-\sqrt{\omega} / 2$, which implies that $E\left(\phi_{\omega}\right) \rightarrow-\infty$ as $\omega \rightarrow \infty$ (see Section 3 below). Clearly, the standing wave solutions exist globally in time. It is an open problem whether the finite time blowing-up occurs or not in this case (see [11]).

Remark 4. In the case of $f(u)=a|u|^{p-1} u$ with $a>0$ and $1<p<5, u_{\omega}$ is stable for any $\omega \in(0, \infty)$. However, Theorem 2 (3) shows that even if $1<p<$ $q<5$, there exist unstable frequencies $\omega$ in the case of $f(u)=a|u|^{p-1} u+b|u|^{q-1} u$ with $a<0, b>0$ and $1<p<q<\infty$. On the other hand, when $a<0, b>0, p=2$ and $q=3$, we can show that $u_{\omega}$ is stable for any $\omega \in(0, \infty)$ (see Section 3 below). Therefore, in Theorem 2 (3), the condition that $p+q>6$ is needed, although it may be not optimal.

\section{Proof of Theorems}

In this section, we prove Theorems 1,2 and 3 stated in Section 1 by using the following lemmas due to Grillakis, Shatah and Strauss [8] and Iliev and Kirchev [9].

Lemma 1. (Grillakis, Shatah and Strauss [8]) Put 


$$
I(\omega)=\left\|\phi_{\omega}\right\|_{L^{2}}^{2}=\int_{-\infty}^{\infty}\left|\phi_{\omega}(x)\right|^{2} d x .
$$

If $I^{\prime}(\omega)>0$, then $u_{\omega}$ is stable, and if $I^{\prime}(\omega)<0$, then $u_{\omega}$ is unstable.

Lemma 2. (Iliev and Kirchev [9]) When $g\left(|u|^{2}\right) u=f(u)=a|u|^{p-1} u+b|u|^{q-1} u$, we have

$$
\begin{aligned}
I^{\prime}(\omega) & =\frac{-1}{2 W^{\prime}(h)} \int_{0}^{h}\left(3-\frac{g(h)-g(s)}{G(h) / h-G(s) / s}\right)\left(\frac{s}{W(s)}\right)^{1 / 2} d s \\
& =\frac{-1}{2 W^{\prime}(h)} \int_{0}^{h} \frac{K(h)-K(s)}{G(h) / h-G(s) / s}\left(\frac{s}{W(s)}\right)^{1 / 2} d s,
\end{aligned}
$$

where $G(s)=\int_{0}^{s} g(\sigma) d \sigma=(2 a / p+1) s^{(p+1) / 2}+(2 b / q+1) s^{(q+1) / 2}, W(s)=\omega s-G(s), K(s)$ $=(a(5-p) / p+1) s^{(p-1) / 2}+(b(5-q) / q+1) s^{(q-1) / 2}$ and $h=h(\omega)$ is a positive number such that $W(h)=0, W^{\prime}(h)<0$ and $W(s)>0$ for any $s \in(0, h)$.

Since it follows from the definition of $h=h(\omega)$ that $G(h) / h-G(s) / s=\omega-$ $G(s) / s>0$ for any $s \in(0, h)$, if $K(h)>K(s)$ for any $s \in(0, h)$, then we have $I^{\prime}(\boldsymbol{\omega})>0$, and if $K(s)>K(h)$ for any $s \in(0, h)$, then we have $I^{\prime}(\omega)<0$.

Put $L(s)=(2 a / p+1) s^{(p-1) i^{2}}+(2 b / q+1) s^{(q-1) i^{2}}$, and let $s_{1}, s_{2}, s_{3}$ and $s_{4}$ be the positive numbers, if they exist, such that $L\left(s_{1}\right)=0, L^{\prime}\left(s_{2}\right)=0, K\left(s_{3}\right)=0$ and $K^{\prime}\left(s_{4}\right)$ $=0$, respectively. That is,

$$
\begin{array}{rlrl}
s_{1}^{(q-p) / 2} & =-\frac{a(q+1)}{b(p+1)}, & s_{2}^{(q-p) / 2} & =-\frac{a(q+1)(p-1)}{b(p+1)(q-1)}, \\
s_{3}^{(q-p) / 2} & =-\frac{a(q+1)(5-p)}{b(p+1)(5-q)}, & s_{4}^{(q-p) / 2}=-\frac{a(q+1)(p-1)(5-p)}{b(p+1)(q-1)(5-q)} .
\end{array}
$$

For the sake of simplicity, we write $K(\infty)$ for $\lim _{s \rightarrow \infty} K(s), h(0)$ for $\lim _{\omega \rightarrow 0}$ $h(\omega)$, and so on.

Proof of Theorem 1. (1) Since $K^{\prime}(s)>0$ for $s>0$, we have $K(h)>K(s)$ for any $s \in(0, h)$, which implies that $I^{\prime}(\omega)>0$ for any $\omega \in(0, \infty)$. Thus, (1) follows from Lemma 1.

(2) Similar to (1).

(3) In this case, we see that $K(0)=0, K^{\prime}(s)>0$ for $s<s_{4}$ and $K^{\prime}(s)<0$ for $s>s_{4}$. Thus, if $h<s_{4}$, then we get $K(h)>K(s)$ for any $s \in(0, h)$, and if $h>s_{3}$, then we have $K(s)>K(h)$ for any $s \in(0, h)$. Moreover, since $L^{\prime}(s)>0$ for $s>0$, $L(0)=0$ and $L(\infty)=\infty$, we see that $h^{\prime}(\omega)>0$ for $\omega>0, h(0)=0$ and $h(\infty)=\infty$. Therefore, (3) follows from Lemma 1.

Proof of Theorem 2. Since $L(0)=0, L^{\prime}(s)<0$ for $s<s_{2}$ and $L^{\prime}(s)>0$ for $s>s_{2}$, we see that $h^{\prime}(\boldsymbol{\omega})>0$ for $\omega>0, h(0)=s_{1}$ and $h(\infty)=\infty$.

(1) When $p \leqq 5 \leqq q$, it follows from $K^{\prime}(s)<0$ for $s>0$ that $K(s)>K(h)$ for 
any $s \in(0, h)$. Thus, we get $I^{\prime}(\omega)<0$ for any $\omega \in(0, \infty)$. When $p>5$, since $K(0)=0, K^{\prime}(s)>0$ for $s<s_{4}$ and $K^{\prime}(s)<0$ for $s>s_{4}$ and $s_{3}<s_{1}$, we have $K(s)>$ $K(h)$ for any $s \in(0, h)$, which implies $I^{\prime}(\omega)<0$ for any $\omega \in(0, \infty)$. Therefore, (1) follows from Lemma 1.

(2) (3) Let $q<5$. Then, we see that $K(0)=0, K^{\prime}(s)<0$ for $s<s_{4}$ and $K^{\prime}(s)$ $>0$ for $s>s_{4}$. Thus, if $h>s_{3}$, then we have $K(h)>K(s)$ for any $s \in(0, h)$, which implies (2). Moreover, if $p+q>6$, then we see that $s_{1}<s_{4}$. Therefore, if $h<s_{4}$, then we have $K(s)>K(h)$ for any $s \in(0, h)$, which implies (3).

Proof of Theorem 3. Since $L(0)=0, L^{\prime}(s)>0$ for $s<s_{2}$ and $L^{\prime}(s)<0$ for $s>s_{2}$, we see that $h^{\prime}(\omega)>0$ for $\omega \in\left(0, \omega^{*}\right), h(0)=0$ and $h\left(\omega^{*}\right)=s_{2}$, where $\omega^{*}=$ $L\left(s_{2}\right)$.

(1) When $p \leqq 5 \leqq q$, it follows from $K^{\prime}(s)>0$ for $s>0$ that $K(h)>K(s)$ for any $s \in(0, h)$. Thus, we get $I^{\prime}(\omega)>0$ for any $\omega \in\left(0, \omega^{*}\right)$. When $q<5$, since $K(0)=0, K^{\prime}(s)>0$ for $s<s_{4}$ and $K^{\prime}(s)<0$ for $s>s_{4}$ and $s_{2}<s_{4}$, we have $K(h)>$ $K(s)$ for any $s \in(0, h)$, which implies $I^{\prime}(\omega)>0$ for any $\omega \in\left(0, \omega^{*}\right)$. Therefore, (1) follows from Lemma 1.

(2) Let $p>5$. Then, we see that $K(0)=0, K^{\prime}(s)<0$ for $s<s_{4}$ and $K^{\prime}(s)>0$ for $s>s_{4}$. Thus, if $h<s_{4}$, then we have $K(s)>K(h)$ for any $s \in(0, h)$. Moreover, since $s_{3}<s_{2}$, if $h>s_{3}$, then we have $K(h)>K(s)$ for any $s \in(0, h)$, which implies (2).

\section{Remarks on special cases}

In this section, we give the proof of the following facts stated in Remarks 3 and 4.

THEOREM 4. If $a<0, b>0, p=2$ and $q=3$, then $u_{\omega}$ is stable for any $\omega \in$ $(0, \infty)$.

Theorem 5. When $a=4, b=3, p=3$ and $q=5$, we have $E\left(\phi_{\omega}\right)=$ $\tan ^{-1}((\sqrt{1+\omega}-1) / \sqrt{\omega})-\sqrt{\omega} / 2$ and $I(\omega)=2 \tan ^{-1}((\sqrt{1+\omega}-1) / \sqrt{\omega}) . \quad$ In particular, we have $E\left(\phi_{\omega}\right) \rightarrow-\infty$ and $I(\omega) \rightarrow \pi / 2$ as $\omega \rightarrow \infty$. Here, we note that $\int_{-\infty}^{\infty}|\tilde{\phi}(x)|^{2} d x$ $=\pi / 2$, where $\tilde{\phi}$ is a positive solution of

$$
-\phi_{x x}+\phi-3 \phi^{5}=0, \quad x \in \boldsymbol{R}, \phi \in H^{1}(\boldsymbol{R}) .
$$

When $q=2 p-1$, the unique solution $\phi_{\omega}$ of (1.2) can be expressed as follows:

$$
\phi_{\omega}(x)=\left(\frac{\omega}{\alpha+\sqrt{\alpha^{2}+\beta \omega} \cosh ((p-1) \sqrt{\omega x}}\right)^{1 /(p-1)},
$$

where $\alpha=a /(p+1)$ and $\beta=b / p$.

Thus, we have 


$$
\begin{aligned}
I(\omega) & =\int_{-\infty}^{\infty}\left|\phi_{\omega}(x)\right|^{2} d x \\
& =\frac{1}{p+1} \int_{-\infty}^{\infty} \frac{|\alpha|^{2 /(p-1)-1}|\beta|^{1 / 2-2 /(p-1)} \nu^{2 /(p-1)-1 / 2}}{(\alpha /|\alpha|+\sqrt{1+(\beta /|\beta|) \nu} \cosh x)^{2 /(p-1)}} d x,
\end{aligned}
$$

where $\nu=\left(|\beta| / \alpha^{2}\right) \omega$.

Proof of Theorem 4. Put

$$
M(\mu)=\int_{-\infty}^{\infty} \frac{\left(\mu^{2}-1\right)^{3 / 2}}{(-1+\mu \cosh x)^{2}} d x,
$$

where $\mu=\sqrt{1+\nu}$. Then, $M(\mu)$ can be calculated as follows:

$$
M(\mu)=4 \tan ^{-1} \sqrt{\frac{\mu+1}{\mu-1}}+2 \sqrt{\mu^{2}-1} .
$$

Thus, we have $M^{\prime}(\mu)=2 \sqrt{\mu^{2}-1} / \mu>0$ for all $\mu>1$. It follows from (3.2) that $I^{\prime}(\omega)>0$ for any $\omega \in(0, \infty)$. Hence, from Lemma 1 , the proof is completed.

Proof of Theorem 5. Since it follows from (1.2) that $-(1 / 2)\left|\phi_{\omega}^{\prime}(x)\right|^{2}+$ $(\omega / 2)\left|\phi_{\omega}(x)\right|^{2}-F\left(\left|\phi_{\omega}\right|\right)(x)=0$ for any $x \in \boldsymbol{R}$, we get

$$
E\left(\phi_{\omega}\right)=\int_{-\infty}^{\infty}\left|\phi_{\omega}^{\prime}(x)\right|^{2} d x-\frac{\omega}{2} \int_{-\infty}^{\infty}\left|\phi_{\omega}(x)\right|^{2} d x
$$

where $\phi_{\omega}^{\prime}(x)=(d / d x) \phi_{\omega}(x)$.

An elementary calculation shows that

$$
I(\omega)=\int_{-\infty}^{\infty}\left|\phi_{\omega}(x)\right|^{2} d x=2 \tan ^{-1}((\sqrt{1+\omega}-1) / \sqrt{\omega}) .
$$

On the other hand, from (3.1), we have

$$
\phi_{\omega}^{\prime}(x)=-\omega \sqrt{1+\omega} \sinh (2 \sqrt{\omega} x)(1+\sqrt{1+\omega} \cosh (2 \sqrt{\omega} x))^{-3 / 2} .
$$

Thus, an elementary computation shows

$$
\int_{-\infty}^{\infty}\left|\phi_{\omega}^{\prime}(x)\right|^{2} d x=(1+\omega) \tan ^{-1}((\sqrt{1+\omega}-1) / \sqrt{\omega})-\sqrt{\omega} / 2
$$

Therefore, from (3.3), (3.4) and (3.5), we obtain

$$
E\left(\phi_{\omega}\right)=\tan ^{-1}((\sqrt{1+\omega}-1) / \sqrt{\omega})-\sqrt{\omega} / 2 .
$$

Acknowledgements. The author would like to express his deep gratitude to Professor Yoshio Tsutsumi for his helpful discussions and advice. He also wishes to express his sincere appreciation to Professor Hayato Nawa for having a special interest in this work and for his encouragement. 


\section{REFERENCES}

[1] I. V. Barashenkov, A.D. Gocheva, V.G. Makhankov and I. V. Puzynin, Stability of the soliton-like "bubbles", Physica D, 34 (1989), 240-254.

[2] H. Berestycki AND T. Cazenave, Instabilité des états stationnaires dans les équations de Schrödinger et de Klein-Gordon non linéaires, C.R. Acad. Sci. Paris, 293 (1981), 489-492.

[3] H. Berestycki AND P.-L. Lions, Nonlinear scalar field equations, I, Arch. Rational Mech. Anal., 82 (1983), 313-345.

[4] T. Cazenave and P.-L. Lions, Orbital stability of standing waves for some nonlinear Schrödinger equations, Comm. Math. Phys., 85 (1982), 549-561.

[5] T. Cazenave and F.B. Weissler, The Cauchy problem for the nonlinear Schrödinger equation in $H^{1}$, Manuscripta Math., 61 (1988), 477-494.

[6] J. Ginibre And G. Velo, On a class of nonlinear Schrödinger equations, I, J. Funct. Anal., 32 (1979), 1-32.

[7] R.T. Glassey, On the blowing up of solutions to the Cauchy problem for nonlinear Schrödinger equations, J. Math. Phys., 18 (1977), 1794-1797.

[8] M. Grillakis, J. Shatah and W. A. Strauss, Stability theory of solitary waves in the presence of symmetry, I, J. Funct. Anal., 74 (1987), 160-197.

[9] I. D. ILIEv AND K.P. KIRChev, Stability and instability of solitary waves for one-dimensional singular Schrödinger equations, Differential and Integral Equations. 6 (1993), 685-703.

[10] T. Kato, On nonlinear Schrödinger equations, Ann. Inst. H. Poincaré Phys. Théor., 46 (1987), 113-129.

[11] K. Kurata and T. Ogawa, Remarks on blowing-up of solutions for some nonlinear Schrödinger equations, Tokyo J. Math., 13 (1990), 399-419.

[12] J. Shatah AND W.A. Strauss, Instability of nonlinear bound states, Comm. Math. Phys., 100 (1985), 173-190.

[13] M.I. Weinstein, Nonlinear Schrödinger equations and sharp interpolation est1mates, Comm. Math. Phys., 87 (1983), 567-576.

Department of Mathematical Sciences

UNIVERSITY OF TOKYO

HONGO, TOKYO 113, JAPAN 